\title{
Anabases
}

ANABASES Traditions et réceptions de l'Antiquité

$21 \mid 2015$

Varia

\section{Le siècle de Platon}

\section{Henri-Irénée Marrou}

\section{OpenEdition}

Journals

Édition électronique

URL : http://journals.openedition.org/anabases/5332

DOI : 10.4000/anabases.5332

ISSN : 2256-9421

\section{Éditeur}

E.R.A.S.M.E.

\section{Édition imprimée}

Date de publication : 1 avril 2015

Pagination : 237-244

ISSN : 1774-4296

\section{Référence électronique}

Henri-Irénée Marrou, «Le siècle de Platon », Anabases [En ligne], 21 | 2015, mis en ligne le 01 avril

2018, consulté le 21 octobre 2019. URL : http://journals.openedition.org/anabases/5332 ; DOI :

10.4000/anabases.5332 


\section{MÉLANGES}

\section{LE SIECLE DE PLATON}

\section{A PROPOS D'UN LIVRE RÉCENT'}

Il faut tenir la parution de ces deux volumes pour un événement considérable. Je suppose n'avoir pas besoin de rappeler à nos lecteurs ce qu'est l'auteur : il y a peu, dans le domaine des études classiques, de personnalités de cette taille vivant à l'heure actuelle; on sait quelle est son activité comme philologue, historien, humaniste et penseur, quelle profonde influence il a exercée sur l'érudition allemande par son enseignement et son rayonnement : il avait groupé toute une école autour de lui, qui s'exprimait, en particulier, dans la revue Die Antike (1925 et suiv.). Ce grand esprit, héritier et émule des Rohde et des Wilamowitz, honneur de la science allemande, a été, Jui aussi, victime de la barbarie nouvelle qui a régné sur son pays. Bien qu'il ait, dans une certaine mesure, parfois trop, à notre goût, sacrifié à l'esprit nouveau que les nazis faisaient triompher 2 , il a dû, atteint indirectement par les lois raciales, quitter l'Université de Berlin et chercher refuge aux États-Unis, comme tant d'autres, comme les savants byzantins du xve siècle l'avaient cherché en Italie. C'est de l'Université Harvard que sont signées les préfaces de ces deux volumes, dont le texte allemand est encore inédit et qui paraissent ici dans la traduction anglaise du professeur Gilbert Highet de Columbia.

On sait quelle noble ambition anime l'œuvre de ce grand éducateur qu'est W. Jaeger : humaniste, il ne sépare pas la connaissance de la vie; ses études sur l'histoire spirituelle du monde grec sont pour lui un moyen de travailler au développement de la culture du temps présent. Sa* vocation d'érudit est soutenue par la conviction que notre civilisation, en dépit de l'élargissement de son horizon, reste un secteur de l'aire hellénocentrique (expression de la préface du recueil, au titre significatif, Altertum und Gegenwart, Leipzig, 1920, p. 11, reprise au début du t. I de Paideia). Selon la curieuse formule

1. Werner JAEgER, Paideia, The Ideals of Greek Culture, vol. II et III. Oxford, Blackwell, 1945, 2 vol. in- $8^{\circ}$, xvır-442 et vir- 374 p.; 22 s. 6 d. chacun.

2. Je pense à l'éloge qu'il fait de l'esprit dorien, de son racisme, de ses tendances artistocratiques et guerrières, si marqué dans le $t$. I et dont subsistent ici quelques échos (III, 79, 236). 
qui termine la préface de la deuxième édition de ce volume I, son étude de la paideia grecque est aussi une étude de l'homme grec en tant que paideia, une contribution, que l'auteur estime essentielle, à l'éducation, à la formation, Formung ${ }^{1}$, de l'homme de notre temps, à la prise de conscience de l'idéal de notre propre culture.

Il est facile de définir en quelques mots la méthode et l'originalité de ce grand ouvrage : il représente l'exemple le plus remarquable de ce que j'ai osé appeler " le nouvel esprit historique ${ }^{2}$ ", cette attitude philosophique à l'égard de l'histoire qui, sans mépriser l'histoire "événementielle ", condition première, base indispensable de toute construction, la considère néanmoins un peu comme elle-même fait des sciences auxiliaires. Le nouvel esprit historique s'intéresse avant tout à l'histoire des idées, à une histoire pour qui l'effort scientifique et désintéressé pour dégager du document la réalité du passé est un moyen, une condition, de la reprise d'idées et de valeurs valables par l'homme vivant. C'est sous le patronage de Platon luimême (par exemple dans la synthèse de l'esprit dorien et de l'esprit attique que représentent les interlocuteurs des Lois) que W. Jaeger place (III, 218) "cette attitude philosophique envers l'histoire qui, au départ de faits historiques imparfaits, s'efforce de s'élever, en s'appuyant sur eux, à quelque chose de parfait et d'absolu ", entendez une essence, Wesen, valable pour nous, support de valeur — en l'espèce, ici, la paideia grecque, l'arétè, la valeur spirituelle de l'homme grec, de l'homme tout court (cf. III, 338, n. 29).

Dans cette perspective, l'auteur a renoncé délibérément à une description concrète de l'éducation, des méthodes pédagogiques antiques : il se tient à un degré supérieur d'abstraction, de profondeur, et analyse les idées fondamentales qui sous-tendent cette éducation, son idéal. Le tome I s'achevait sur un beau chapitre sur Thucydide, apogée et crise de l'esprit attique; après dix ans ${ }^{3}$, W. Jaeger nous offre la suite attendue : le siècle de Platon, suite attendue avec impatience; nous savions l'intérêt passionné que l'auteur porte à Platon et les articles-programme parus dans Die Antike en 1928 nous donnaient déjà un aperçu de la thèse de l'auteur (La place de Platon dans l'édification de la culture grecque, notamment le troisième article, $L a$ philosophie platonicienne comme " paideia »). Notre attente n'a pas été déçue : ce n'est pas, comme l'annonçait la préface du tome Ier, un, mais deux gros volumes que W. Jaeger nous donne sur "l'âge de Platon " (ainsi que le sous-titre du tome III définit le Iv $^{\mathrm{e}}$ siècle), disons mieux sur Platon luimême, dont Jaeger célèbre magnifiquement le rôle hors de pair dans le

1. Dans le premier volume, Paideia avait comme sous-titre "La formation, Formung, de l'homme grec $n$.

2. Cf. Dieu vivant, 1945, 3, p. 146-147.

3. La préface du t. I est datée de juillet 1935 ; de la seconde édition allemande (Berlin, 1936) existe une traduction italienne (éd. La Nuova Italia, Florence, 1936) et une traduction anglaise que l'éditeur Blackwell nous annonce en voie de réimpression. 
développement de la pensée et de la tradition grecques et à l'étude duquel il s'attache avec tant de complaisance qu'il finit par faire éclater les cadres primitivement imposés à son cuvre : le lecteur finit par se demander si le véritable sujet du livre n'est pas une initiation à l'étude de Platon et si toute cette "morphologie génétique " de la paideia, de la tradition culturelle grecque, n'est pas simplement amenée à titre "d'indispensable arrière-plan philosophique à la compréhension de Platon " (II, 84).

Oui, en somme, ces deux volumes constituent un Platon analogue comme méthode au beau livre que nous avait déjà donné W. Jaeger sur Aristote ${ }^{1}$; comme celui-ci, nous avons ici " les fondements d'une histoire du développement spirituel " de Platon. Particulièrement heureux est l'effort pour replacer cette évolution intérieure dans son milieu et son temps : A. Rey eût aimé le beau chapitre sur la valeur culturelle de la médecine grecque (III, 3 et suiv.), dont Jaeger montre l'influence profonde, au moins égale à celle des mathématiques, qu'elle a eue sur Platon (idéal de la connaissance efficace, unissant théorie et praxis, schéma méthodologique qui a permis l'élaboration de la théorie des Idées); de même, les chapitres qui suivent, sur Xénophon et Isocrate, intercalés entre l'analyse de la République, d'une part, celle du Phaedre et des Lois, d'autre part, servent-ils à illustrer l'effort toujours repris par, Platon pour assouplir sa doctrine, assimiler toutes les influences. Subtilement, W. Jaeger montre Isocrate, dans l'A Nicolès et l'Antidosis, répondant au Gorgias et, par contre-coup, le Phaedre amené à reconsidérer sur une base nouvelle le problème de la rhétorique; dans ce débat, Platon n'est pas seul : l'auteur souligne l'intérêt de l'œuvre de Speusippe, du rôle du jeune Aristote " privat-docent " de rhétorique à l'Académie. De même, il détecte dans les Lois une critique de la Cyropédie ou une influence du Cynégétique, tandis que, par ailleurs, des Mémorables du même Xénophon contribuent au même mouvement de propagande "réactionnaire " que l'A réopagitique d'Isocrate.

Par de telles observations se dessine peu à peu devant nous un tableau remarquablement précis et nuancé du Zeitgeigst qui fait de ce livre une contribution remarquable à l'histoire de cette période attachante (à mesure qu'elle nous est mieux connue) qu'est le Iv siècle. L'historien, j'entends celui qui s'intéresse d'abord à l'histoire politique, aura, en particulier, beaucoup à relever dans les chapitres consacrés à Isocrate (III, 46 et suiv.) : celui-ci nous apparaît comme le porte-parole de la nouvelle droite, disons de la droite constitutionnelle modérée, pour qui le grand homme fut et resta l'élève préféré d'Isocrate, Timothée, fils de Conon, que Jaeger oppose, dans une formule heureuse, au "battle-scarred swashbuckler " Charès, "l'idole du parti radical " (III, 139). Reprenant les conclusions de son mémoire des Harvard Studies de 1941, W. Jaeger date l'A réopagitique d'avant (et non, comme d'autres, d'après) les désastres de la guerre sociale (357-

1. Aristoteles, Grundlegung einer Geschichte seiner Entwtcklung. Berlin, 1923. 
$355)$ : ce discours prend ainsi la valeur d'un avertissement prophétique que le parti de Timothée, alors écarté des affaires, adresse à la politique de ses successeurs incompétents (III, 109-110). Cette droite modérée, dont Platon et Aristote incarnent eux aussi, par moments, la position, tire les leçons de la chute de la Seconde Confédération athénienne et propose une politique de repliement, l'abandon de toute politique de constructions navales et d'interventions lointaines (III, 239). Quant au stade final de la carrière d'Isocrate, à la "trahison " qui le fait collaborer avec Philippe, Jaeger n'hésite pas à en faire l'apologie : entre la Macédoine et Isocrate se noue un front commun de résistance spirituelle : si l'essence du génie national d'Athènes c'est la culture, la paideia, la Macédoine était l'alliée désignée pour sauver celle-ci, compromise par les démocrates grossiers, défenseurs de la cause non seulement perdue, mais inutile, de la petite polis (III, 152-154).

Enfin, le chapitre final, qui fait un peu hors d'œuvre, sur Démosthène, sera à l'usage un résumé commode de la thèse soutenue par Jaeger dans son livre de $1938^{1}$ et qui, réagissant contre la dépréciation systématique dont la mémoire du grand orateur est l'objet en Allemagne depuis Droysen, vise à le réhabiliter en l'éclairant par une connaissance plus précise de son temps : Démosthène apparaît comme l'héritier des leaders de la Seconde Confédération, ces impérialistes assagis par l'amère expérience de la génération de la défaite, et notamment de Callistrate, "clear headed and strongwilled's; Démosthène appartient à ses débuts au milieu d'Eubule, à cette tendance néo-conservatriçe qui s'exprime, par ailleurs, dans le discours $S u r$ la Paix d'Isocrate et le traité des Revenus de Xénophon. Peu à peu, il élargit l'ambition de ses discours, dont Jaeger célèbre la portée éducative : dans cette démocratie à gouvernement direct, abandonnée aux mauvais bergers, il n'y a de salut que dans un effort pour éduquer le peuple lui-même, l'amener à la maturité politique. Apologie nuancée, sans illusion : le souvenir de Démosthène apparaît à Jaeger comme enveloppé de mélancolie, triste comme les visages de Scopas...

Si intéressantes que soient ces pages, elles ne viennent qu'en supplément : l'essentiel de l'effort est consacré, je l'ai dit, à Platon. Sur ce sujet, l'auteur s'est épanoui, s'attardant à plaisir à relire devant nous ces belles œuvres. Une à une, il les analyse, égrenant au passage ses réflexions et ses commentaires. La lecture en est un peu ralentie (l'analyse devient parfois si détaillée, par exemple pour les livres VIII-IX de la République, qu'elle devient traduction) et on s'impatiente : le lecteur d'aujourd'hui est toujours si pressé! Jaeger est un humaniste.

II nous donne donc ici son Platon : une grande synthèse analogue à celle de Wilamowitz. Analogue et rivale : e'est l'adversaire vis-à-vis de qui Jaeger sent le besoin de se poser - de s'opposer (ainsi II, 88, 93, 96...). De

1. Demosthenes, The Origin and Growth of his Policy. Berkeley, 1938. 
façon générale, Jaeger ne s'attarde pas en polémiques de détail : soucieux de se situer dans le courant de l'histoire de l'interprétation de la pensée platonicienne (forme moderne de la permanence du platonisme même), il se contente pourtant de se définir vis-à-vis de quelques grands noms, Schleiermacher, Gomperz, Wilamowitz. Comme il est de tradition chez les savants allemands, Jaeger ne s'intéresse guère à l'érudition étrangère (sinon à l' "école écossaise ": Burnet, Taylor), ne cite guère qu'en passant Diès, Rey ou Gilson et ignore les synthèses platoniciennes de Festugière ou Moreau. Aussi retrouve-t-il pour son compte bien des jdées déjà familières au lecteur français : nous connaissions par P. Boyancé la portée de la législation des banquets et des fêtes dans les Lois (ici III, 223 et suiv., 226 et suiv.), par le P. des Places la disqualification, en Tyrtée, de l'idéal archaïque, ou spartiate, de la vertu guerrière (III, 221, 230).

Mais on ne peut demander à un savant de la classe de Jaeger de s'astreindre, tel un candidat au doctorat, à se situer minutieusement vis-à-vis de ses prédécesseurs : il nous apporte ici le résultat d'une longue méditation, est son exposé, dégagé de tout débat, fonde sa conviction moins sur une discussion minutieuse d'arguments que sur l'impression d'ensemble que laisse à l'auteur une longue expérience. Méthode évidemment dangereuse, que seul un maître peut se permettre et qui, même en de si bonnes mains, ne laisse pas de donner l'impression de l'arbitraire. Ainsi, le chapitre initial sur Socrate nous surprend un peu par l'assurance avec laquelle il trie chez Platon ce qui est proprement platonicien et ce qui doit être compté à Socrate (ainsi II, 42, 49, 151, 154, 304). Surtout après que Jaeger lui-même eût remarquablement analysé l'ambiguité essentielle, que dis-je le caractère insoluble, du problème : il faut bien que Socrate ait été lui-même ambigu pour avoir donné naissance à l'agnosticisme d'Antisthène aussi bien qu'au dogmatisme de Platon; et l'effort même que nous tcntons pour dégager Platon de Socrate est condamné par Platon lui-même, qui n'a jamais consenti à se penser distinct de l'influence que Socrate a eue sur lui (II, 19). Mais nous n'allons pas soulever à nouveau tout le problème de la structure logique de la certitude en histoire : dans ce domaine, où l'épistémè épidictique est inaccessible, il faut se résigner à demeurer au niveau de la doxa. Nous n'avons ici qu'une opinion sur Platon; c'est au moins, je le répète, celle d'un maitre, qui parle au nom de toute son autorité.

Il est difficile d'analyser la richesse de ees deux volumes. Si Jaeger enrichit l'histoire de préoccupations philosophiques, il reste bien un historien par son sens du réel, du concret : Platon ne se dissout pas en un réseau d'opinions abstraites, il reste devant nous un homme. D'où des remarques qu'aucun philosophe de profession, pour qui le système importe plus que la personne, n'aurait su faire (sur la signification, par exemple, du réalisme platonicien : là théorie des Idées est antérieure aux découvertes d'Aristote sur le concept : II, 101 et suiv, ; ou encore sur Platon comme prédécesseur de Freud : II, 343). 
S'il s'oppose si consciemment à Wilamowitz, c'est que, parti du même point de départ (l'authenticité des lettres VI-VIII) et mû par la même ambition (décrire l'itinéraire spirituel de Platon), W. Jaeger atteint un résultat différent. Alors que Wilamowitz, plus littéraire, plus biographe, n'hésitait pas à morceler l'œuvre du maitre, ne voyant, par exemple, dans les dialogues socratiques que des œuvres de jeunesse, simples exercices littéraires sans apport philosophique positif, Jaeger souligne l'unité profonde de tout Platon. Son héros nous apparait, un peu comme le César de J. Carcopino, comme un personnage très unifié, remarquablement conscient de lui-même, possédant, jeune encore, une vue arrêtée sur ce que devait être l'œuvre de sa vie. "Quand il écrivait les premiers mots de son premier dialogue socratique, il savait déjà ce que serait l'ensemble où il viendrait s'insérer. L'entéléchie de la République se dessine déjà clairement dans ces premiers dialogues " (II, 96).

Réagissant contre la tendance littéraire de Wilamowitz à voir dans la succession des dialogues un reflet de l'évolution psychologique de Platon et comme une " confession lyrique ", W. Jaeger souligne leur caractère objectif, resserre la trame qui les unit : des jalons posés dans le Protagoras amorcent le Gorgias; le Gorgias, à son tour, annonce la République comme le Ménon enchaine avec Protagoras. La succession des œuvres engendre un système : avec, bien entendu, un art consommé, sans rien qui sente l'application mécanique d'un programme à priori : l'œuvre unique et variée grandit sous nos yeux " avec la liberté et l'assurance d'un puissant arbre " (II, 96) - ou, si on préfère, d'une courbe savante dont chaque nouveau segment, une fois construit, amène à reconsidérer l'ensemble.

La destinée de Platon se joua dans le choc psychologique qu'a représenté pour le jeune aristocrate ambitieux de politique la rencontre de Socrate; la conversion morale qu'il effectue l'amène non à renoncer à la politique, mais à placer à la base de celle-ci la vertu, ou mieux, comme Jaeger nous invite à traduire l'intraduisible aretè, la " perfection spirituelle ". Rejeté dans la pensée pure par le malheur des temps (de la mort de Socrate aux désillusions et aux tragédies de Sicile), Platon reste fidèle à cette double ambition : une politique saine fondée sur la vertu. D'où son idée maitresse (que Jaeger reproche amèrement à Gomperz d'avoir méconnue) : l'État, pour être fondé sur la vertu, doit se faire éducateur, sa fonction essentielle étant non la conquête de la puissance, mais d'élever les citoyens, et d'abord leurs chefs, à la perfection morale. C'est pourquoi l'œuvre centrale de Platon est la République, et la République est avant tout un traité d'éducation (sa cité idéale étant un État artificiellement imaginé pour le mettre en mesure de remplir sa tâche éducative). Jaeger reprendrait volontiers à son compte le titre du livre de J. Stenzel, Plato der Erzieher (1938), Platon l'Éducateur. A ses yeux, la pédagogie n'est pas un aspect, fût-il privilégié, de son cuvre : elle constitue l'essence même du génie platonicien.

Point de vue original, remarquable - évidemment violemment orienté, 
comme le faisceau de lumière d'un projecteur qui fouille profondément l'objet dans une direction propre, laissant le reste dans l'ombre. Si cette hypothèse rend compte de la place éminente qu'occupent la République et les Lois dans l'œuvre de Platon et celle que les problèmes d'éducation prennent dans ces chartes constitutionnelles, elle néglige les dialogues que d'autres lecteurs, plus métaphysiciens qu'humanistes, revendiqueront comme essentiels. Je ne parle pas du Timée, que la synthèse de Jaeger ne réussit qu'imparfaitement à intégrer (il s'en excuse : III, 346, n. 215), mais du Théetète, du Parménide ou du Sophiste, qu'elle abandonne tout à fait, quitte à les qualifier dédaigneusement de "sèches investigations méthodologiques »(II, 176).

Point de vue, donc, nécessairement partiel, partial. Fécond, cependant : encore une fois, je renonce à en énumérer les aperçus ingénieux; l'analyse de Jaeger aboutit à souligner le caractère profondément religieux de la pensée platonicienne : pensée "extatique " où c'est Dieu, et non plus l'homme, qui devient la mesure de toutes choses (Lois, 716 b : ici II, 287 ; III, 241). De façon un peu paradoxale, Jaeger formule son jugement en concluant que l'éducation platonicienne apparaît comme une anticipation prophétique de ce que devait devenir... le catholicisme (III, 252); traduisons : parti pour construire une cité, Platon aboutit à rêver d'une Église ; Plotin n'avait donc pas si tort à rêver d'une Platonopolis qui eût été une espèce de couvent ${ }^{1}$...

Mais point de vue passionné : comme pour Ch. Picard, l'art grec, et peutêtre l'art humain, trouve son apogée et sa justification dernière en Phidias ; la culture grecque, pour Jaeger, atteint avec Platon sa forme suprême. Cette décision l'amène à un véritable parti pris : son Isocrate, si richement dessiné, si fouillé soit-il, est en définitive délibérément sacrifié à Platon (III, 69, 148).

On hésitera à ratifier toujours ce jugement. Dire que Platon est « le vrai

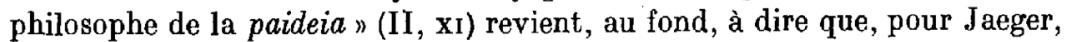
le message platonicien est celui-là même que la culture moderne a surtout besoin d'écouter. Mais je ne sais pas s'il ne se laisse pas trop séduire par l'évidente puissance de cette haute pensée. Je crois possible d'envisager le devenir de la paideia antique sous un autre éclairage. Il faut revenir ici sur le fait que la place démesurée accordée par notre auteur à Platon a fait éclater le plan qu'il se proposait d'abord de suivre : Jaeger nous annonce sans doute un volume ultérieur sur les destinées de la culture à l'époque hellénistique ${ }^{2}$, mais, à voir comment déjà il dénonce chez Aristote " un

1. Gf. E. Brénter, Introduction à son édition des Ennéades de Plotin, p. Xilı.

2. Il voudrait même aller plus loin et poursuivre jusqu'à l'époque chrétienne (n'oublions pas que W. Jaeger avait assumé la direction de l'édition critique de saint Grégoire de Nysse), mais il n'ose trop préciser sa promesse : ce grand travailléur atteint et mesure les limites de la vie humaine; il jette dans une note (II, 414, n. 39 b) l'esquisse d'une Théologie de Platon, d'une suite à sa Théologie des philosophes grecs archaïques (Gifford Lectures de St. Andrews, 1936, actuellement sous presse), suite qu'il n'est pas sưr de pouvoir donner un jour. 
remarquable déclin d'intensité " dans la conception de la paideia, je ne suis pas sûr qu'il soit en mesure de lui rendre pleine justice et d'y voir autre chose qu'une lente décadence.

Alors qu'au contraire je situerais volontiers à l'époque hellénistique (prolongée jusqu'à la fin du Haut-Empire romain : je crois à l'unité de l'hellenistisch-römische Kultur) l'akmè de la paideia antique, l'apogée de l'art de modeler la forme intérieure de la personnalité humaine. Par contre-coup, j'en viendrais à revaloriser, vis-à-vis de Platon, le rôle historique d'Isocrate, le grand initiateur au point de vue des institutions pédagogiques, le fondateur de la grande tradition de l'école antique. Car, historiquement, PIaton est un vaincu ; ce n'est pas lui qui a été suivi par les générations d'après; disons que l'ambition si haute et si pure qui l'animait l'a empêché de voir les limites de la nature humaine, de répondre aux aspirations réelles de l'humanité de son temps. Philosophiquement, et je dirai même humainement, Isocrate ne peut être mis en parallèle avec Platon, qui le dépasse de cent coudées. Historiquement, sociologiquement, son rôle a été bien autrement considérable. Platon avait trop attendu de l'homme : son rêve quasi messianique d'un philosophe-roi est demeuré sans réalisation. Isocrate, pius terre à terre, a couru au plus pressé : faire des hommes, hic et nunc, et il a jeté les bases d'une pédagogie dont toute une civilisation, la civilisation antique, dont toute une tradition, la tradition classique, ont vécu, dont nous vivons encore.

Sans doute est-ce là aussi un point de vue, tout aussi passionné et partiel que celui de W. Jaeger. Je ne suis pas sûr d'être en mesure de jamais le défendre avec autant de puissance persuasive et de fécondité que W. Jaeger l'a fait pour le sien.

H.-I. Marrou.

Professeur à la Sorbonne. 